\title{
LA COMPRENSIÓN DE LAS ORACIONES DE RELATIVO EN ESPAÑOL: LOS EFECTOS DE LA INTERVENCIÓN SINTÁCTICA EN LAS RELATIVAS SEMILIBRES Y EN LAS ENCABEZADAS
}

\author{
Marisol Murujosa \\ Universidad de Buenos Aires / Consejo Nacional de Investigaciones Científicas y Técnicas - CONICET \\ (Argentina) \\ marisolmurujosa@conicet.gov.ar \\ Carolina Gattei \\ Pontificia Universidad Católica Argentina / Universidad Torcuato Di Tella / Universidad de Buenos Aires \\ (Argentina) \\ carolina.gattei@conicet.gov.ar \\ Diego Shalom \\ Universidad de Buenos Aires / Universidad Torcuato Di Tella / Consejo Nacional de Investigaciones Científicas \\ y Técnicas - CONICET (Argentina) \\ diegoshalom@gmail.com \\ Yamila Sevilla \\ Universidad de Buenos Aires / Consejo Nacional de Investigaciones Científicas y Técnicas - CONICET \\ (Argentina) \\ ysevilla@filo.uba.ar
}

Recibido: 14/08/2020 - Aprobado: 18/09/2020 - Publicado: 15/04/2021

DOI: doi.org/10.17533/udea.lyl.n79a05

Resumen: Se llevó a cabo un estudio de comprensión auditiva de oraciones de relativo (OR) encabezadas y semilibres en la variante del español argentino. Los resultados muestran que las ORs de objeto son más difíciles de comprender que las de sujeto, tanto en el caso de las encabezadas como en el de las semilibres. Estos resultados argumentan a favor de la propuesta de intervención por inclusión de rasgos morfosintácticos (Rizzi, 2004), ya que en ambos tipos de ORs de objeto se encuentra un restrictor léxico, propiedad expresada por un rasgo nominal $[+\mathrm{N}]$, que interfiere en el establecimiento de la dependencia sintáctica.

Palabras clave: comprensión de oraciones; oraciones relativas; intervención sintáctica; relativas encabezadas; relativas semilibres.

\section{COMPREHENSION OF RELATIVE CLAUSES IN SPANISH: SYNTACTIC INTERVENTION ON HEADED AND FALSE FREE RELATIVES}

Abstract: In this study, an auditory comprehension task of headed and false free relative clauses (RC) in Argentinean Spanish is presented. The results show that in both cases object RCs were more difficult to comprehend than subject ORs. This fact is consistent with the proposal of syntactic intervention by inclusion of morphosyntactic features (Rizzi, 2004). Both, headed and semi-free object RCs, are lexically restricted by a lexical element with the feature $[+\mathrm{N}]$ and this element interferes in the establishment of the syntactic dependency.

Key words: sentence comprehension; relative clauses; syntactic intervention; headed relatives; false free relatives.

Editores 


\section{Introducción}

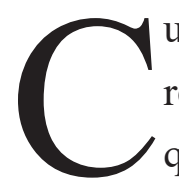

uando se comprende la oración «El senador al que le gritó el periodista es alto», se necesita establecer una relación entre el elemento nominal senador y la posición de objeto del verbo gritar para poder interpretar que fue el senador quien recibió una réplica acalorada. Esa conexión representa una dependencia de larga distancia (DLD), en la que un elemento se pronuncia en una posición estructuralmente distante a la posición original en la que recibió su rol temático. Las oraciones de relativo (OR), las preguntas qu-, las oraciones topicalizadas, son ejemplos de estructuras con DLD.

Las DLD son, entonces, las relaciones sintáctico-semánticas entre un elemento desplazado (filler) y su posición sintáctica original (gap). Es por este motivo que las oraciones con DLD han sido un fenómeno privilegiado para estudiar la relación entre los mecanismos sintácticos y la dificultad de procesamiento y, por lo tanto, han brindado un campo muy fértil para la investigación psicolingüística.

\subsection{Las oraciones de relativo en español: encabezadas, libres y semilibres}

Este estudio se centra en la comprensión de una clase particular entre las estructuras con DLD: las ORs especificativas, como las que se observan en (1). En español, las ORs son oraciones subordinadas encabezadas por un pronombre, adjetivo o adverbio relativo que funcionan como complementos modificadores de un elemento, llamado «antecedente» (Brucart, 1999), habitualmente nominal.

(1) a. Relativa encabeZada de sujeto

$\mathrm{El}\left[\mathrm{OR}_{\mathrm{O}}\right.$ senador que __saludó al periodista] es joven.

b. Relativa encabezada DE OBJeto

El [ ${ }_{\mathrm{OR}}$ periodista al que saludó el senador__ ] es joven.

En oraciones como las de (1) se ha propuesto (Vergnaud, 1974; Kayne, 1994; Bianchi, 1999) que el sintagma nominal (SN) antecedente de la relativa se genera dentro de la OR, en la posición de gap indicada en (1) con guiones bajos, y se desplaza hacia la periferia oracional (filler). El antecedente de las ORs es típicamente un nombre o un sintagma nominal léxico.

A su vez, existen construcciones en las que la OR modifica a un elemento que no tiene realización fonológica, como ocurre en (2). Estas, conocidas como ORs libres, están formadas por un pronombre relativo, cuyo antecedente se considera nulo (Di Tullio, 2005, p. 312). En este tipo de estructuras es el pronombre relativo el que se desplaza a la periferia oracional.

(2) a. ReLATIVA LIBRE DE SUJETO 
$\mathrm{Vi}$ a [ ${ }_{\mathrm{OR}}$ quien _ te saludó ayer].

b. RELATIVA LIBRE DE OBJETO

$\mathrm{Vi}\left[{ }_{\mathrm{OR}}\right.$ a quien saludaste _ ayer].

En español existe, además, un tercer tipo de OR, cuyo antecedente no tiene contenido fonológico, pero que sí se encuentra en la estructura sintáctica: las ORs semilibres. Estas oraciones se caracterizan por poseer un antecedente nominal tácito, cuya presencia se evidencia en los rasgos de flexión y de número del determinante. Se ha propuesto — p. ej. Giollo y Muñoz Pérez (2013), y, desde la perspectiva de la morfología distribuida, Kornfeld y Saab (2005) y Saab (2009) — que las ORs semilibres poseen la misma estructura sintáctica que las encabezadas, con la excepción de que en las primeras el elemento que se desplaza a la periferia oracional es un nombre nulo, elemento nuclear listado en el léxico (Panagiotidis, 2003).

(3) a. ReLATIVA SEMILIBRE DE SUJETO

$\mathrm{El}\left[\mathrm{OR}_{\mathrm{OR}} \mathrm{N}_{\text {nulo }}\right.$ que _ le gritó al periodista] saludó al senador.

b. RELATIVA SEMILIBRE DE OBJETO

El senador saludó al [ ${ }_{\mathrm{OR}} \mathrm{N}_{\text {nulo }}$ que le gritó el periodista _ $]$.

Por lo tanto, el objetivo de este trabajo es estudiar la comprensión de este tercer tipo de DLD, poco estudiada en la bibliografía psicolingüística.

\subsection{La asimetría de sujeto/objeto en la comprensión de las oraciones relativas}

Diversos estudios con distintas poblaciones, técnicas y tareas han demostrado, por ejemplo, que a diferencia de las ORs de sujeto (ORS) encabezadas, las ORs de objeto (ORO) encabezadas son más difíciles de producir y comprender, se adquieren más tardíamente y dan lugar a rendimientos más deficientes en personas con afasia. La comprensión de ORs, en particular, es un fenómeno ampliamente estudiado.

La asimetría entre la comprensión de ORSs y la de las OROs es, de hecho, uno de los resultados más consistentes en la literatura sobre procesamiento. Estos datos, que representan un conjunto asombrosamente homogéneo, se obtuvieron en niños y en adultos en diferentes lenguas, como el español (Betancort et al., 2009), inglés (Brown, 1972; Frazier et al., 1983; Gibson, 1998), italiano (Contemori \& Belletti, 2014), hebreo (Friedmann et al., 2009), chino (Hu et al., 2016), griego (Theodorou \& Grohmann, 2012). Estos efectos se hacen presentes también en el rendimiento de personas con afasia (Garraffa \& Grillo, 2008; Grillo, 2009; Sánchez et al., 2017), y en niños con TEL, dislexia y otros trastornos del lenguaje (Adani et al., 2014, Cantiani et al., 2013; Casalis et al., 2013; Arosio et al., 2017).

La propuesta de Minimidad Relativizada por Rasgos, (fRM por sus siglas en inglés), (Rizzi, 2004) argumenta que la asimetría en el procesamiento de ORs encabezadas evidenciada en diversas lenguas es consecuencia de un 
fenómeno denominado intervención sintáctica por inclusión de rasgos morfosintácticos. En ese sentido, Friedmann et al. (2009) argumentaron que la similitud estructural entre el sujeto subordinado y el objeto, antecedente nominal, dificulta la comprensión de OROs con dos SNs animados, como las oraciones de (1) retomadas en (4). Por ende, los dos SNs son estructuralmente similares, ya que ambos contienen un elemento nominal explícito, i.e., una «restricción léxica», propiedad expresada por un rasgo [+N].

\section{(4) a. Relativa ENCABEZADA DE SUJETO}

El $\left[_{\mathrm{OR}}\right.$ señador ${ }_{[\mathrm{N}+\mathrm{R}]}$ que _[N ${ }_{[\mathrm{N}]}$ saludó al periodista $\left.{ }_{[\mathrm{N}]}\right]$ es joven.

b. RELATIVA ENCABEZADA DE OBJETO

El [ ${ }_{\mathrm{OR}}$ per Todista $_{[\mathrm{N}+\mathrm{R}]}$ al que saludó el senador $\left.\left.{ }_{[\mathrm{N}]}\right]_{\mathrm{R}}\right]$ es joven.

En el caso de las OROs (4b), el restrictor léxico en el sujeto (senador) subordinado interviene en el desplazamiento del antecedente nominal (periodista) desde la posición en la que se interpreta como objeto del verbo subordinado (saludar) hacia la periferia oracional. Entonces, según Friedmann et al. (2009), pero cf. también Grillo (2009), el SN nominal sujeto, al ser estructuralmente similar, interfiere en el establecimiento de la dependencia sintáctica entre filler y gap y en la correcta interpretación de la oración. En cambio, el caso de las ORSs (4a), no hay ningún elemento que interfiera entre filler y gap y el procesamiento se facilita.

Asimismo, Friedmann et al. (2009) argumentaron que si el restrictor léxico con el rasgo [+N] no se manifiesta en alguno de los constituyentes involucrados en el establecimiento de esta dependencia sintáctica, la intervención no se produciría y el procesamiento de la oración debería facilitarse, aún en el caso de OROs. Para sostener esta hipótesis, los autores manipularon la aparición del restrictor léxico y evaluaron la comprensión de ORs libres de sujeto (5a) y de objeto (5b) en niños hablantes de hebreo, en las que el elemento desplazado consiste en un pronombre relativo.

(5)

a. Tare li et mi she-martiv et ha-yeled. mostra a-mí ACC quien que-moja ACC el-niño

"Mostrame quien moja al niño".

b. Tare li et mi she-ha-yeled menadned. mostra a-mí ACC quien que-el-niño hamaca.

"Mostrame a quien hamaca el niño".

Nota: Tomados de Friedmann et al. (2009, pp.73-74).

Los resultados mostraron que la comprensión de OROs por parte de los niños mejoraba significativamente 
Los efectos de la intervención sintáctica en las relativas semilibres y en las encabezadas

cuando el restrictor léxico no estaba presente en uno de los elementos involucrados en el establecimiento de la dependencia, i.e., en el caso de las ORs libres. En concreto, los autores afirmaron que esto se debe a que el target del movimiento y el interventor eran morfosintácticamente disímiles, esto es, solo uno estaba léxicamente restringido, es decir, poseía el rasgo [+N].

\subsection{Las relativas semilibres en español: los restrictores léxicos nulos}

Como se señaló anteriormente, las ORs semilibres en español se caracterizan por poseer un restrictor léxico nulo y no ausente, como es el caso de las ORs libres. Pero, a diferencia de las OR encabezadas, en las semilibres el restrictor léxico carece de contenido fonológico. En este sentido, las ORs semilibres permiten poner a prueba las predicciones de la fRM. Si la intervención depende de la presencia de un restrictor léxico pleno con realización fonológica, como ocurre con las ORs encabezadas, el procesamiento de las ORs semilibres, que carecen de este elemento, se verá facilitado y, por lo tanto, no se observará la asimetría ORO/ORS típica, en línea con los hallazgos de Friedmann et al. (2009) con las ORs libres. Si, en cambio, como predice la fMR, la intervención depende de la presencia sintáctica del restrictor léxico, aunque este sea nulo y sin contenido fonológico, las OROs semilibres deberían generar los mismos efectos de intervención y ser, por lo tanto, más difíciles de procesar que las ORSs semilibres.

Para evaluar la comprensión de ORs semilibres y compararlas con ORs encabezadas, se diseñó una tarea de comprensión auditiva. Las variables independientes que se consideraron fueron el tipo de OR (ORS u ORO) y tipo de antecedente (encabezadas o semilibres). Las variables dependientes fueron la tasa de acierto en las respuestas de los participantes y el tiempo de respuesta (TR).

\section{Experimento}

\subsection{Metodología}

\subsubsection{Participantes}

Un total de 33 sujetos (25 mujeres y 8 hombres) formaron parte del experimento. Todos los participantes eran hablantes nativos de español de Argentina, de entre 18 y 35 años de edad $(M=24.39, E S=0.74)$, con visión normal o corregida a normal y con agudeza auditiva normal. Ninguno de los sujetos reportó enfermedades neurológicas, abuso de drogas o de alcohol, desórdenes psiquiátricos, trastornos específicos del lenguaje o problemas de aprendizaje. Todos los participantes dieron su consentimiento por escrito previamente a realizarse el experimento. 


\subsubsection{Materiales}

Los estímulos auditivos de este experimento consistieron en oraciones relativas construidas a partir de una lista de 20 verbos agentivos que marcan con caso nominativo al agente y con caso dativo al objeto, que es el beneficiario del evento, ${ }^{1}$ por ejemplo, sonreír. Se controló la frecuencia en base logarítmica según la base de datos EsPal (Duchon et al., 2013), $M=1.97, E S=0.43$. Las listas de verbos pueden consultarse en el Anexo 1.

Para construir las ORs se seleccionaron 40 sustantivos (36 profesiones y 4 animales) con los que se armaron 20 parejas con el mismo género morfológico (10 parejas con género masculino y 10 parejas con género femenino) que se pueden consultar en el Anexo 2.

La variable que se manipuló para construir las ORs fue el tipo de antecedente (encabezadas o semilibres). A su vez, se manipuló el tipo de relativa (de sujeto o de objeto) y se crearon dos versiones de las oraciones, alternando el rol de los participantes. De esta manera, cada verbo se presentó en ocho condiciones:

ORDEN DE PART.
TIPO
P1 (PAYASO) - P2 (BEBÉ)
P2 (BEBÉ) - P1 (PAYASO)

DE RELATIVA

\begin{tabular}{ccc}
\hline EncABEZADA DE SUJETo & $\begin{array}{c}\text { En la imagen aparece el } \\
\text { payaso que le sonríe al bebé. }\end{array}$ & $\begin{array}{c}\text { En la imagen aparece el bebé que le } \\
\text { sonríe al payaso. }\end{array}$ \\
\hline EnCABEZADA DE OBJETO & $\begin{array}{c}\text { En la imagen aparece el } \\
\text { payaso al que le sonríe el } \\
\text { bebé. }\end{array}$ & $\begin{array}{c}\text { En la imagen aparece el bebé al que } \\
\text { le sonríe el payaso. }\end{array}$ \\
\hline SEMILIBRE DE SUJETO & $\begin{array}{c}\text { En la imagen aparece el que } \\
\text { le sonríe al bebé. }\end{array}$ & $\begin{array}{c}\text { En la imagen aparece el que le } \\
\text { sonríe al payaso. }\end{array}$ \\
\hline SEMILIBRE DE OBJETO & En la imagen aparece al que & En la imagen aparece al que le \\
le sonríe el bebé. & sonríe el payaso.
\end{tabular}

Tabla 1. Ejemplo de cada condición experimental con el verbo sonreír

Las 80 oraciones resultantes fueron grabadas y editadas en PRAAT (Boersma \& Weenink, 2019) y se dividieron en ocho listas balanceadas de acuerdo con el diseño de cuadrado latino, de manera que cada sujeto solo escuchó una versión de las ocho oraciones construidas con cada verbo. Dentro de cada lista el orden de presentación de los estímulos fue aleatorio, así como el carácter verdadero o falso de cada oración en relación a la imagen que se les presentaba; cada sujeto escuchó la mitad de las oraciones verdaderas y la otra mitad falsas.

Las 40 imágenes del experimento fueron dibujos en blanco y negro de los 40 eventos en las que también 1. alteruaban tos roles de los participantes, A su vez, se usaron dos imágenes espejadas de cada. una de estas 800 comportamiento sintáctico, por fuera de la marcación de caso, es equivalente al de los verbos con objetos acusativos. 


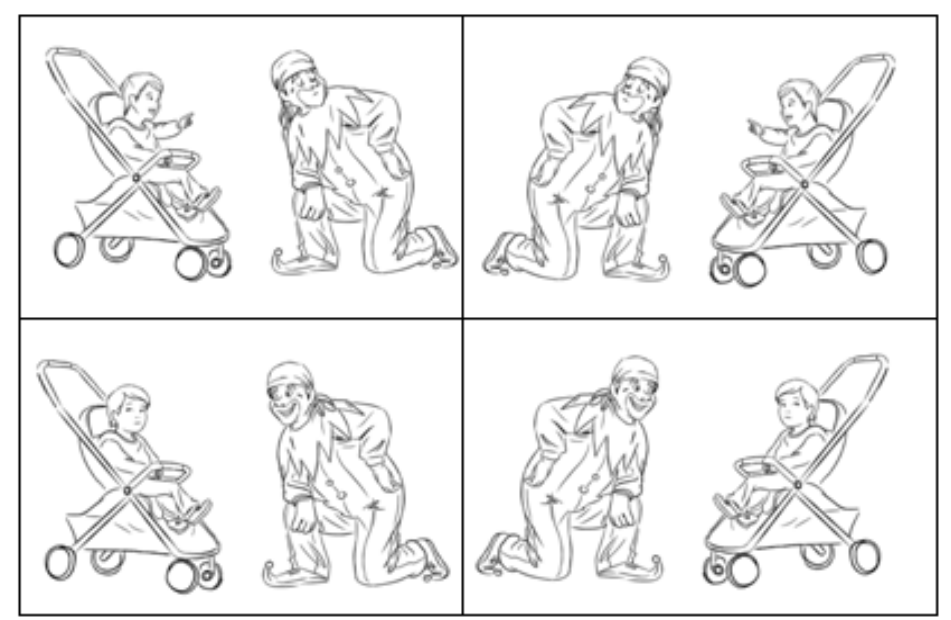

Imagen 1. Material visual del presente experimento de comprensión: sonreír

Además, se crearon 4 estímulos de práctica, así como 40 estímulos que se utilizaron como distractores. Estas oraciones se diferenciaban de los estímulos blancos en complejidad sintáctica y longitud, para que los participantes no se percataran del objetivo del experimento, por ejemplo: En la imagen aparece una persona recibiendo un premio.

Para controlar la aceptabilidad de los estímulos blancos se llevó a cabo una tarea de juicios de gramaticalidad en línea. En esta tarea participaron un total de 177 participantes hablantes nativos de la variante de español de Argentina, de entre 18 a 69 años de edad $(M=32.68 ; E S=0.17)$. A los participantes se les pidió que puntuaran en una escala Likert del 1 al 7 la aceptabilidad una serie de oraciones, entre las que se encontraban los cuatro tipos de oraciones blanco (Tabla 1), oraciones agramaticales como controles y oraciones diversas como distractores. Una prueba estadística de muestras independientes no paramétrica de Kruskal-Wallis reveló que había diferencias significativas entre los tipos de oraciones $\left(X^{2}=754.8 ; D F=4 ; p<.001\right)$. Un análisis de comparaciones múltiples posthoc mostró que había diferencias significativas entre oraciones agramaticales y oraciones blanco (el valor de $p$ fue $<.001$ en las cuatro condiciones). Este análisis mostró también que las OROs semilibres se comportaron de una manera diferente al resto de las condiciones blanco. Es decir, fueron significativamente peor puntuadas que el resto de las ORs (el valor de $p$ de cada comparación con las otras tres condiciones fue $<.001$ ).

\subsubsection{Procedimiento}

El inicio del trial comenzaba cuando los sujetos posaban su mirada en la cruz de fijación que se encontraba en el centro de la pantalla. $2000 \mathrm{~ms}$ después se reproducía el audio de la oración. La cruz de fijación se mantenía en la pantalla por $500 \mathrm{~ms}$ desde la finalización del audio y luego aparecía la imagen. Los participantes debían decidir si 


\section{Los efectos de la intervención sintáctica en las relativas semilibres y en las encabezadas}

la oración que habían escuchado era verdadera o falsa en relación con la imagen que se les presentaba después. Si la oración era verdadera, debían apretar la flecha derecha en el teclado; si la oración era falsa, la flecha izquierda. Luego de que apretaran la tecla, la imagen desaparecía y se les volvía a mostrar la pantalla en blanco por 2000 ms.

\subsubsection{Análisis de datos}

El análisis de los datos fue realizado a través de la plataforma de programación R (R CoreTeam, 2020), principalmente mediante el paquete «lme4» (Bates et al., 2015). Un Modelo Lineal de Efectos Mixtos (MLEM) fue ajustado a los datos de TRs y un Modelo Lineal Generalizado de Efectos Mixtos (MLGEM) con una función binomial, a los datos dicotómicos de la tarea de comprensión.

Se tomaron el Rendimiento o los TRs como variables dependientes, el Tipo de Antecedente (Encabezadas vs. Semilibres) y el Tipo de Relativa (ORS vs. ORO) como variables fijas, la Frecuencia del Verbo como variable de control y Sujeto e Ítem como variables aleatorias. Los modelos también incorporaron la interacción entre las dos variables fijas.

Se incluyeron estructuras de efectos aleatorios máximos; cuando los modelos no convergían o la correlación entre la varianza de los componentes no podía ser estimada, se removían las correlaciones para simplificar la estructura de efectos aleatorios. Para muestras de datos como la obtenida, la distribución de $t$ se aproxima a la distribución normal, y un valor absoluto del valor de $t$ o $z$ mayor a 2 indica un efecto significativo al valor de $\alpha=$ 0,05 .

Para el análisis de TRs, se transformaron los tiempos en milisegundos a valores en escala logarítmica (log), ya que los TRs en escala de segundos o milisegundos pueden conducir a conclusiones incorrectas dada la falta de distribución normal de sus residuos (ver Bayen y Milin (2010), para una discusión sobre este tema). Sin embargo, para mejorar la comprensión de los datos, los TRs fueron reportados y graficados en segundos (s). Para el análisis se tomaron en cuenta solo los TRs de las respuestas correctas de los participantes.

\subsection{Resultados}

\subsubsection{Rendimiento}

Los participantes respondieron de forma correcta el $86 \%$ de los estímulos $(E S=1.4 \%)$. En la Figura 1 se pueden observar las medias y los errores estándar obtenidos para cada tipo de condición. En el caso de ORs encabezadas, hubo un $95 \%(E S=1.7 \%)$ de respuestas correctas a los estímulos con ORSs y un $79 \%(E S=3.2$ \%) a los estímulos con OROs. En el caso de ORs semilibres, el porcentaje de respuestas correctas fue de un $91 \%$ $(E S=2.2 \%)$ para los estímulos con ORSs y de $79 \%(E S=3.2 \%)$ para los estímulos con OROs. 
Los efectos de la intervención sintáctica en las relativas semilibres y en las encabezadas

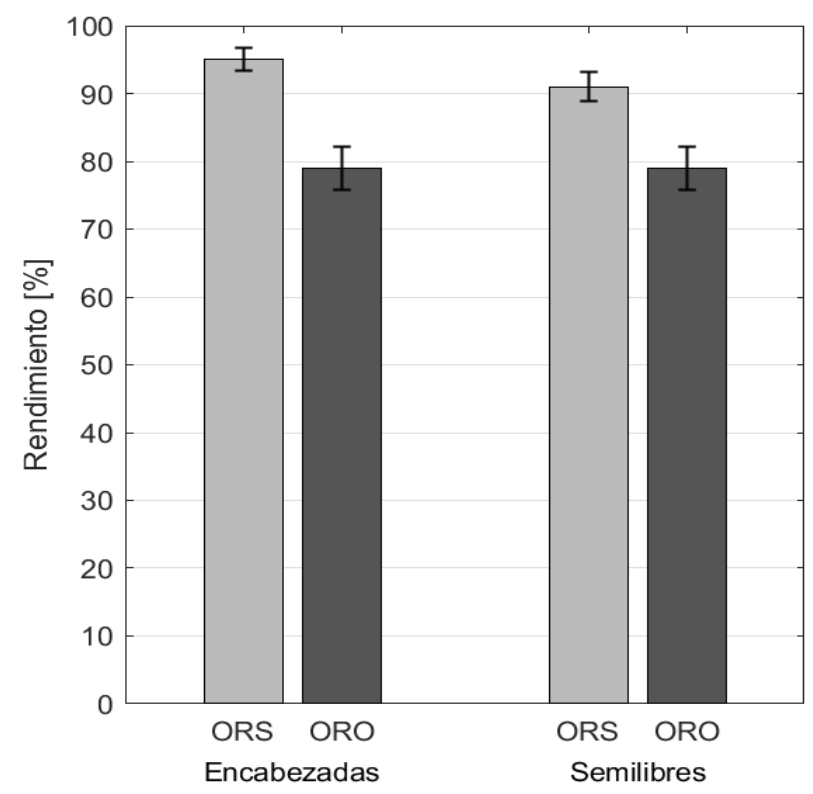

Figura 1. Porcentaje de aciertos promedio $( \pm E S)$ de los participantes en la tarea de comprensión. ORS = Oración Relativa de Sujeto; ORO = Oración Relativa de Objeto

Se utilizó un MLGEM para predecir el porcentaje de respuestas correctas de acuerdo con el tipo de antecedente y el tipo de relativa. Se encontró un efecto principal de tipo de relativa $(\beta=0.83, E S=0.18, z=4.54, p<.001)$, pero no se encontró un efecto principal de Tipo de Antecedente $(\beta=-0.16, E S=0.17, z=-0.90, p=0.37) \mathrm{y}$ tampoco se encontró una interacción significativa entre ambas variables $(\beta=-0.20, E S=0.17, z=-1.18, p=0.24)$.

\subsubsection{Tiempos de respuesta}

El tiempo de respuesta total promedio fue de $2.33 \mathrm{~s}(E S=0.05 \mathrm{~s})$. En la Figura 2 se pueden observar las medias y los errores estándar obtenidos para cada condición. En el caso de ORs encabezadas, el TR promedio fue $2.05 \mathrm{~s}(E S=0.08 \mathrm{~s})$ en los estímulos con ORSs y $2.58 \mathrm{~s}(E S=0.12 \mathrm{~s})$ en estímulos con OROs. En el caso de ORs semilibres, el TR promedio fue $2.15 \mathrm{~s}(E S=0.08 \mathrm{~s})$ en los estímulos con ORSs y $2.63 \mathrm{~s}(E S=0.12 \mathrm{~s})$ en estímulos con OROs. 


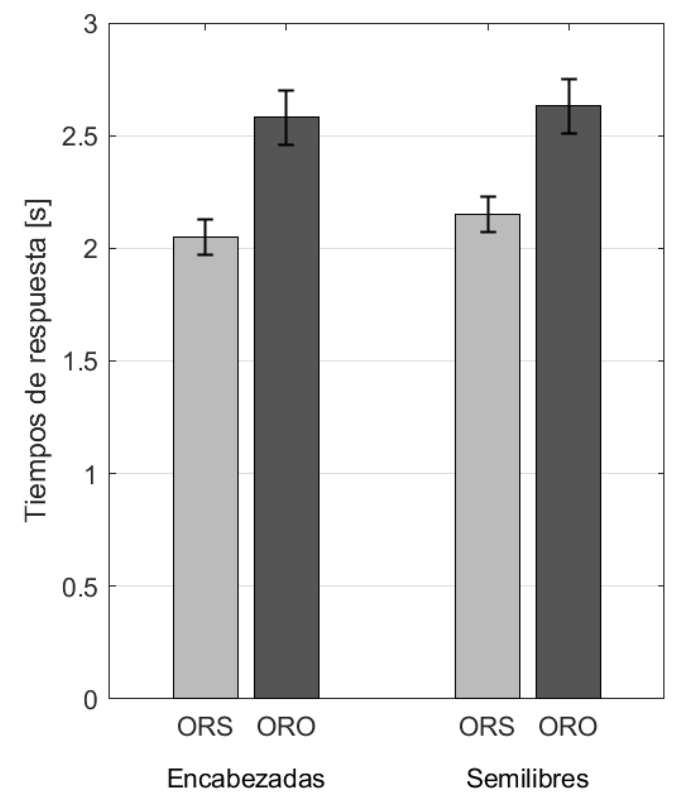

Figura 2. Porcentaje de TRs ( $\pm E S)$ de los participantes en la tarea de comprensión. ORS = Oración Relativa de Sujeto; ORO = Oración Relativa de Objeto

El análisis de las diferencias en TRs de acuerdo con el Tipo de Relativa y el Tipo de Antecedente reveló efectos significativos solo del Tipo de Relativa $(\beta=-0,09, E S=0,02, t=5.71, p<.001)$. Es decir, no se encontró un efecto principal de Tipo de Antecedente $(\beta=0.02, E S=0.02, t=1.26, p=0.21)$ y tampoco se encontró una interacción significativa entre ambas variables $(\beta=0.003, E S=0.02, t=0.45, p=0.66)$.

\section{Discusión}

En la presente investigación se propuso indagar si, en el caso de las OROs semilibres, la presencia de un restrictor léxico nulo sin realización fonética genera los mismos efectos de intervención sintáctica que han sido documentados para las OROs encabezadas. Siguiendo la propuesta de fRM, se predijo que la presencia sintáctica del restrictor léxico es suficiente para generar la intervención, aunque este elemento no tenga contenido fonológico.

El análisis de los resultados muestra que las OROs fueron significativamente más difíciles de comprender - mayor tasa de error y mayores latencias en las respuestas - que las ORSs, independientemente de si estas eran encabezadas o semilibres. En ambos casos, los resultados obtenidos son consistentes con la hipótesis de intervención sintáctica por inclusión de rasgos propuesta por fRM. Para las ORs encabezadas, el establecimiento de la dependencia sintáctica entre filler y gap se dificulta cuando hay un elemento interventor (6b) y esto se traduce en una disminución en el rendimiento de los participantes. En términos de fRM, el SN sujeto interfiere en el desplazamiento del SN objeto a la periferia. Si ese elemento interventor no aparece, como en las ORSs (6a), no 
se manifiestan efectos de intervención.

(6)

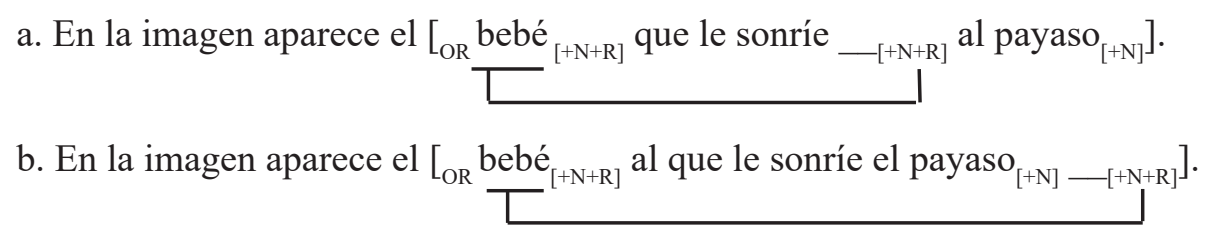

En las OROs semilibres (7b), se da la misma configuración con intervención sintáctica por inclusión de rasgos que en las encabezadas (6b), aunque el restrictor léxico sea un nombre nulo sin contenido fonológico. Respecto a las ORSs semilibres (7a), como no hay ningún elemento interventor entre filler y gap, estas son comprendidas más fácilmente.

(7)

a. En la imagen aparece el $\left[\mathrm{OR}_{\mathrm{On}} \mathrm{N}_{\text {nulo[N] }}\right.$ que le sonríe __

b. En la imagen aparece al que $\left[\mathrm{OR}_{\text {nulo[N+R] }}\right.$ le sonríe el payaso $\left.{ }_{[\mathrm{N}]} \mathrm{N}_{[\mathrm{N}+\mathrm{R}]}\right]$.

Es por eso que los resultados obtenidos muestren los mismos efectos de intervención para ambos tipos de OROs, tanto en las encabezadas como en las semilibres, y que no se haya encontrado un efecto significativo de la variable Tipo de Antecedente. Estos hallazgos son consistentes con la propuesta de fRM, esto es, que la intervención se da por la inclusión de rasgos morfosintácticos debido a la presencia de un restrictor léxico y el rasgo $[+\mathrm{N}]$, independientemente de que dicho restrictor léxico tenga contenido fonológico o no.

Sin embargo, como se mencionó previamente, los estímulos con OROs semilibres utilizadas en el experimento muestran cierta degradación en relación al resto de las ORs. Una posible explicación para este resultado es el hecho de que se haya utilizado el verbo aparecer como verbo de la oración matriz. En el caso de las OROs semilibres (7b) el verbo aparecer esta seguido inmediatamente por la marca de caso dativo a que corresponde al objeto desplazado hacia la periferia, lo que genera cierta incongruencia en la asignación de caso nominativo por parte del verbo de ascenso aparecer. Aunque es poco probable que esa degradación dé cuenta de los resultados obtenidos, sería interesante explorar el comportamiento de las OR semilibres introducidas por predicados con diferente marcación de caso para separar la potencial influencia de este factor de los efectos de intervención.

La presente investigación se enmarca dentro de un amplio grupo de estudios que buscan indagar de qué manera las particularidades de las distintas lenguas y sus propiedades gramaticales repercuten en diversos fenómenos de procesamiento, particularmente en el caso de oraciones con DLD. En este sentido, la propuesta de fRM ha permitido explicar diversos fenómenos de comprensión y producción del lenguaje en distintas lenguas y poblaciones, al llevar la atención a las diferentes configuraciones sintácticas subyacentes y a los rasgos morfosintácticos activos 
Los efectos de la intervención sintáctica en las relativas semilibres y en las encabezadas

en cada uno de los elementos involucrados en el establecimiento de la DLS, por ejemplo, los rasgos de animacidad, género, número y caso, y que también han sido considerados responsables de la intervención en algunos casos (Belletti et al. 2012; Friedmann, Belletti \& Rizzi, 2016; Adani et al., 2010, 2014, 2017; Bentea, Durrleman \& Rizzi, 2016; Manetti et al. 2016; Corrêa, Rodrigues \& Forster, 2019; Shetreet \& Friedmann, 2014; Terzi \& Nanousi, 2018; Adelt et al. 2017, entre otros).

No obstante, las condiciones consideradas en la presente investigación no permiten discernir si la intervención se genera por la presencia del restrictor léxico o simplemente la presencia del rasgo [+N], aun en ausencia del restrictor léxico. En esta línea, Costa, Grillo y Lobo (2012) estudiaron la comprensión de OR libres en portugués europeo y encontraron efectos de intervención en las OROs libres. Los autores argumentan que esto se debe a que en portugués europeo el pronombre relativo quem posee entre sus rasgos a los rasgos [+N], [+animado], [+humano], aunque no haya en la estructura un restrictor léxico nominal, y que es la presencia de estos rasgos la que genera la intervención sintáctica. Es por este motivo que proponemos en el futuro estudiar la comprensión de ORs libres en español, ya que permitirían continuar ajustando la propuesta teórica de fRM en relación al procesamiento de oraciones con DLD.

\section{Conclusiones}

Estudios previos en diversas lenguas, incluido el español, han evidenciado mayores dificultades en el procesamiento de oraciones relativas de objeto con restrictor léxico, i.e., encabezadas. Algunos estudios, sin embargo, reportaron que esta dificultad no se encuentra en el caso de oraciones relativas sin restrictor léxico, como el caso de las ORs libres en hebreo (Friedmann et al., 2009). La comprensión de ORs semilibres de sujeto y de objeto en español que poseen restrictor léxico mas no contenido fonológico no había sido estudiada hasta el momento. En la presente investigación, se estudió si la presencia de un restrictor léxico nulo sin contenido fonológico, en el caso de las OROs semilibres, podía dar lugar a los mismos efectos de intervención observados en el caso de las OROs encabezadas — p.ej. en español, Betancort et al. (2009).

En efecto, en este estudio, las OROs semilibres mostraron idénticas dificultades en el procesamiento (mayores errores y mayores latencias) que las OROs encabezadas. Dichas dificultades pueden ser explicadas por los efectos de la intervención de un elemento en el establecimiento de la DLD. En ambos casos, el SN sujeto interfiere en el desplazamiento del SN objeto hacia la periferia, ya que ambos son estructuralmente similares, ambos están restringidos léxicamente y poseen el rasgo [+N]. Esta inclusión de rasgos morfosintácticos dificulta el establecimiento de la dependencia y la correcta interpretación de la oración.

Particularmente, en las ORs semilibres el objeto desplazado contiene un restrictor léxico, y por ende el rasgo [+N], ya que están formadas por un elemento nominal nulo seleccionado léxicamente (Giollo \& Muñoz Pérez, 2013). Se mostró así que los efectos de intervención no dependen de la presencia explícita, con realización fonética, del restrictor léxico. Tal como lo propone la fRM, la presencia en la configuración sintáctica, aún sin 
Los efectos de la intervención sintáctica en las relativas semilibres y en las encabezadas realización fonética, de un restrictor léxico da lugar a interferencia en el establecimiento de las DLD.

En futuros estudios, se propone investigar, por un lado, de qué manera repercuten en el procesamiento de ORs en español las posibles diferencias entre los rasgos morfosintácticos (género, número y caso) de los SNs involucrados en el establecimiento de la DLD, así como la ausencia de un restrictor léxico en uno de los elementos, como en el caso de las ORs libres. 


\section{Referencias bibliográficas}

1. Adani, F., Van der Lely, H. K., Forgiarini, M., \& Guasti, M. T. (2010). Grammatical Feature Dissimilarities Make Relative Clauses Easier: A Comprehension Study with Italian Children. Lingua, 120(9), 2148-2166. doi: 10.1016/j.lingua.2010.03.018

2. Adani, F., Forgiarini, M., Guasti, M. T., \& Van Der Lely, H. K. (2014). Number Dissimilarities Facilitate the Comprehension of Relative Clauses in Children

with (Grammatical) Specific Language Impairment. J. Child Lang., 41, 811-841. doi: 10.1017/S0305000913000184 3. Adani, F., Stegenwallner-Schütz, M., \& Niesel, T. (2017). The Peaceful Co-Existence of Input Frequency and Structural Intervention Effects on the Comprehension of Complex Sentences in German-Speaking Children. Frontiers in Psychology, 8. doi: 10.3389/fpsyg.2017.01590

4. Adelt, A., Stadie, N., Lassotta, R., Adani, F., \& Burchert, F. (2017). Feature Dissimilarities in the Processing of German Relative Clauses in Aphasia. Journal of Neurolinguistics, 44, 17-37. doi: 10.1016/j.jneuroling.2017.01.002 5. Arosio, F., Panzeri, F., Molteni, B., Magazù, S., \& Guasti, M. T. (2017). The Comprehension of Italian Relative Clauses in Poor Readers and in Children with Specific Language Impairment. Glossa J. Gen. Linguist., 2(9). doi: http://doi.org/10.5334/gjgl.107

6. Baayen, H. R. \& Milin, P. (2010). Analyzing Reaction Times. International Journal of Psychological Research, 3(2), 12-28. doi: 10.21500/20112084.807

7. Bates, D., Maechler, M., Bolker, B., \& Walker, S. (2014). lme4: Linear Mixed-Effects Models Using Eigen and S4, package R version 1.1-7. http:// CRAN.R-project.org/package=lme4

8. Belletti, A., Friedmann, N., Brunato, D., \& Rizzi, L. (2012). Does Gender Make a Difference? Comparing the Effect of Gender on Children's Comprehension of Relative Clauses in Hebrew and Italian. Lingua, 122(10), 10531069. doi: 10.1016/j.lingua.2012.02.007

9. Bentea, A., Durrleman, S., \& Rizzi, L. (2016). Refining Intervention: The Acquisition of Featural Relations in Object A-Bar Dependencies. Lingua, 169, 21-41. doi: 10.1016/j.lingua.2015.10.001

10. Betancort, M., Carreiras, M., \& Sturt, P. (2009). The Processing of Subject and Object Relative Clauses in Spanish: An Eye-Tracking Study. Quarterly Journal of Experimental Psychology, 62(15), 1915-1929. doi: $10.1080 / 17470210902866672$

11. Bianchi, V. (1999). Consequences of Antisymmetry: Headed Relative Clauses. Berlin: Mouton De Gruyter. doi: $10.1515 / 9783110803372$

12. Boersma, P. \& Weenink, D. (2019). Praat: Doing Phonetics by Computer, version 6.0.50. http://www.praat. org.

13. Brown, H. (1972). Children's Comprehension of Relativized English Sentences. Child Development, 42 , 1923-1936.

14. Brucart, J. M. (1999). La estructura del sintagma nominal: las oraciones de relativo. En V. Demonte \& I. 
Los efectos de la intervención sintáctica en las relativas semilibres y en las encabezadas

Bosque (Coords.), Gramática descriptiva de la lengua española, Vol. 1 (pp. 395-522). Madrid: Espasa-Calpe.

15. Cantiani, C., Lorusso, M. L., Guasti, M. T., Sabisch, B., \& Männel, C. (2013). Characterizing the Morphosyntactic Processing Deficit and its Relationship to Phonology in Developmental Dyslexia. Neuropsychologia, 51, 1595 1607. doi: 10.1016/j.neuropsychologia.2013.04.009

16. Casalis, S., Leuwers, C., \& Hilton, H. (2013). Syntactic Comprehension in Reading and Listening: A Study with French Children with Dyslexia. Journal of Learning Disabilities, 46, 210-219. doi: 10.1177/0022219412449423 17. Contemori, C. \& Belletti, A. (2014). Relatives and Passive Object Relatives in Italian-Speaking Children and Adults: Intervention in Production and Comprehension. Applied Psycholinguistics, 35, 1021-1053. doi: 10.1017/ s0142716412000689

18. Corrêa, L., Rodrigues, E., \& Forster, R. (2019). On the Processing of Object Relative Clauses, 57-60. ExLing 2019: Proceedings of 10th International Conference of Experimental Linguistics, 25-27 September 2019, Lisbon, Portugal. doi: 10.36505/ExLing-2019/10/0014/000376

19. Costa, J., Grillo, N., \& Lobo, M. (2012). Minimality Beyond Lexical Restrictions: Processing and Acquisition of Free WH-Dependencies in European Portuguese. Revue Roumaine de Linguistique, 57.

20. Di Tullio, Á. (2005). Manual de gramática del español. Buenos Aires: La Isla de la Luna.

21. Duchon, A., Perea, M., Sebastián-Gallés, N., Martí, A., \& Carreiras, M. (2013). EsPal: One-Stop Shopping for Spanish Word Properties. Behavior Research Methods, 45(4), 1246-1258.

22. Frazier, L., Clifton, C., \& Randall, J. (1983). Filling Gaps: Decision Principles and Structure in Sentence Comprehension. Cognition, 13(2), 187-222. doi: 10.1016/0010-0277(83)90022-7

23. Friedmann, N., Belletti, A., \& Rizzi L. (2009). Relativized Relatives: Types of Intervention in the Acquisition of A-Bar Dependencies. Lingua, 119(1), 67-88. doi: 10.1016/j.lingua.2008.09.002

24. Friedmann, N., L. Rizzi, \& A. Belletti. (2016). No Case for Case in Locality: Case Does Not Help Interpretation When Intervention Blocks A-Bar Chains. Glossa, 2(1), 33, 1-18.

25. Garraffa, M. \& Grillo, N. (2008). Canonicity Effects as Grammatical phenomena. Journal of Neurolinguistics, 21, 177-197. doi: 10.1016/j.jneuroling.2007.09.001

26. Gibson, E. (1998). Linguistic Complexity: Locality of Syntactic Dependencies. Cognition, 68, 1-76. doi: 10.1016/S0010-0277(98)00034-1

27. Giollo, N. \& Muñoz Pérez, C. (2013). Sobre las construcciones relativas en español. En A. Marcovecchio, A. Ghío \& M. Cuñarro (Eds.), En torno a la morfosintaxis del español. Mendoza: Universidad Nacional de Cuyo, 49-59.

28. Grillo, N. (2009). Generalised Minimality: Feature Impoverishment and Comprehension Deficits in Agrammatism. Lingua, 119, 1426-1443. doi: 10.1016/j.lingua.2008.04.003

29. Hu, S., Gavarró, A., Vernice, M., \& Guasti, M. T. (2016). The Acquisition of Chinese Relative Clauses: Contrasting Two Theoretical Approaches. Journal of Child Language, 43, 1-21. doi: 10.1017/S0305000914000865 30. Kayne, R. S. (1994). The Antisymmetry of Syntax. Cambridge: MIT Press. 
Los efectos de la intervención sintáctica en las relativas semilibres y en las encabezadas

31. Kornfeld, L. \& Saab, A. (2005). Hacia una tipología de las anáforas nominales en español. III Encuentro de Gramática Generativa, Universidad del Comahue, Neuquén, 18-20 de agosto de 2005.

32. Manetti, C., Moscati, V., Rizzi, L., \& Belletti, A. (2016). The Role of Number and Gender Features in the Comprehension of Italian Clitic Left Dislocations. En Proceedings of the 40th annual Boston University Conference on Language Development [BUCLD40] (pp. 229-240).

33. Panagiotidis, P. (2003). Empty Nouns. Natural Language \& Linguistic Theory, 21, 381-432.doi: 10.1023/A:1023384924981

34. R Core Team (2020). R: A Language and Environment for Statistical Computing. R Foundation for Statistical Computing, Vienna, Austria. https://www.R-project.org/.

35. Rizzi, L. (2004). Locality and the Left Periphery. En Belletti, A. (Ed.), Structures and Beyond: The Cartography of Syntactic Structures, Vol. 3 (pp. 223-251). Oxford/New York: OUP.

36. Saab, A. (2009). Hacía una teoría de la identidad parcial en la elipsis (Tesis doctoral inédita). Buenos Aires: Universidad de Buenos Aires.

37. Sánchez, M. E.; Taboh, A.; Fuchs, M.; Barreyro, J. P. \& Jaichenco, V. (2017). Comprensión de oraciones con cláusulas relativas. Un estudio comparativo entre sujetos con y sin alteraciones del lenguaje. Panamerican Journal of Neuropsychology, 11(3). doi: 10.7714/CNPS/11.3.211.

38. Shetreet, E., \& Friedmann, N. (2014). The Processing of Different Syntactic Structures: fMRI Investigation of the Linguistic Distinction between Wh-Movement and Verb Movement. Journal of Neurolinguistics, 27(1), 1-17. doi: 10.1016/j.jneuroling.2013.06.003

39. Terzi, A. \& Nanousi, V. (2018). Intervention Effects in the Relative Clauses of Agrammatics: The Role of Gender and Case. Glossa: A Journal of General Linguistics, 3(1), 17. doi: 10.5334/gjgl.274.

40. Theodorou, E. \& Grohmann, K. K. (2012). The Acquisition of Relative Clauses in Cypriot Greek: Production and Comprehension. Revista Diacrítica, 26, 270-299.

41. Vergnaud, J. R. (1974). French Relative Clauses (Tesis doctoral no publicada) Cambridge, Massachusetts: MIT. 
Los efectos de la intervencidn Anexo 1: Lista de verbos

\begin{tabular}{|c|}
\hline apuntar \\
\hline cantar \\
\hline chiflar \\
\hline chillar \\
\hline chistar \\
\hline cocinar \\
\hline discutir \\
\hline gritar \\
\hline gruñir \\
\hline guiñar \\
\hline leer \\
\hline murmurar \\
\hline planchar \\
\hline protestar \\
\hline rogar \\
\hline silbar \\
\hline sonreír \\
\hline suplicar \\
\hline susurrar \\
\hline tararear \\
\hline
\end{tabular}

\begin{tabular}{|c|}
\hline Anexo 2: Parejas de participantes \\
\hline bailarina - monja \\
\hline abuela - nieta \\
\hline maestra - bruja \\
\hline fotógrafa - nadadora \\
\hline mendigo - millonario \\
\hline princesa - hada \\
\hline esquiadora - policía \\
\hline violinista - director \\
\hline perro - león \\
\hline enfermero - niño \\
\hline cura - presidente \\
\hline mozo - cocinero \\
\hline mujer - nena \\
\hline luchadora - médica \\
\hline oso - mono \\
\hline reina - hechicera \\
\hline payaso - bebé \\
\hline marinero - soldado \\
\hline árbitro- jugador \\
\hline cantante - pianista \\
\hline
\end{tabular}

